\title{
Targeting mass-selected cluster ions for the deposition of advanced carbonaceous materials using an inductively coupled plasma
}

\author{
D. A. Zeze a) and S. R. P. Silva \\ Electronic Engineering, University of Surrey, Guildford GU2 7XH, United Kingdom
}

\author{
N. M. D. Brown, A. M. Joyce, and C. A. Anderson \\ Surface Science Laboratory, University Of Ulster, Coleraine BT52 1SA, Northern Ireland
}

(Received 5 July 2001; accepted for publication 26 November 2001)

\begin{abstract}
Study has been initiated of the deposition of thin films using cluster ions in differing abundances generated in a pulsed inductively coupled plasma. A pulse unit controlling the "on" and "off" timing ratio of a $13.56 \mathrm{MHz}$ rf power supply is used to alter systematically the composition of the contents of the plasma. Adamantane $\left(\mathrm{C}_{10} \mathrm{H}_{16}\right)$ vapor, in argon or nitrogen, was selected as precursor for the deposition of $\mathrm{C}_{x} \mathrm{H}_{y}$ or $\mathrm{C}_{x} \mathrm{~N}_{y} \mathrm{H}_{z}$ thin films using this pulsed source. The effect of varying the relative abundances of the cluster ions present in the resultant plasmas on the films produced, by changing driving power on/off ratio, is investigated. The mass-energy diagnostic data recorded under 50/150 and 150/50 $\mu$ s on/off pulse sequences showed that fragmentation of $\mathrm{C}_{10} \mathrm{H}_{16}$ is a function of the switching ratio selected, i.e., clusters of different sizes and abundances are thus produced. Langmuir probe data also suggest that the electrical characteristics of the plasma generated under these regimes can be monitored. Postdeposition x-ray photoelectron spectroscopy (XPS) analysis of specimen films deposited under the same on/off regimes, within a $200 \mu$ s cycle, indicate clear differences are present in these films in terms of the atomic concentration, the $\mathrm{C} 1 \mathrm{~s}$ envelope bandwidths, and profiles. Contact angle measurements and optical data performed were found to reflect the variations in the differently deposited films already indicated in the XPS data. For example, specimens deposited under a 50/150 on/off ratio show a high contact angle (low surface energy) and lower refractive index, whereas those deposited under a 150/50 ratio exhibit a low contact angle (high surface energy). Films prepared using the same precursor streams but with the further on/off ratios, 20/180, 100/100, and 20/180 $\mu$ s, were also considered. Postdeposition analyses confirmed the same tends in the fragmentation behavior of $\mathrm{C}_{10} \mathrm{H}_{16}$. Finally, the significance of such a cluster ions selection technique is discussed. (C) 2002 American Institute of Physics.
\end{abstract}

[DOI: $10.1063 / 1.1435417]$

\section{INTRODUCTION}

Different carbon and hydrocarbon sources have been used as precursors for the growth of diamond-like amorphous carbon (DAC) and hydrogenated carbon nitride $\left(\mathrm{C}_{x} \mathrm{~N}_{y} \mathrm{H}_{z}\right)$ films with the ultimate aim of synthesizing wear resistant films which offer good chemical, optical, mechanical, and tribological properties. ${ }^{1}$ Traditionally, graphite and low molecular weight hydrocarbons $\left(\mathrm{C}_{1} \mathrm{H}_{x}\right.$ and $\left.\mathrm{C}_{2} \mathrm{H}_{x}\right)$ have been the main carbon sources used for the deposition of diamond, DAC and $\mathrm{C}_{x} \mathrm{~N}_{y} \mathrm{H}_{z}$ films deposited using various plasma regimes. In this context, higher molar mass molecules such as adamantane, $\mathrm{C}_{10} \mathrm{H}_{16}(136.24 \mathrm{MW})$, are of interest in that these offer the possibility of deposition via $\mathrm{C}_{x} \mathrm{H}_{y}$ clusters of significant size, ${ }^{2}$ where $x=3-10$. An important potential benefit in this case is that carbon clusters are reported ${ }^{3}$ to play a significant role in the growth of diamond, DAC, and related CN films. However, tuning selectively ${ }^{4}$ cluster ions has still to be addressed. Note, there are many reports dealing with either high ${ }^{5,6}$ mass carbon

\footnotetext{
a) Author to whom correspondence should be addressed; electronic mail: daz_ze@hotmail.com
}

clusters and $\operatorname{low}^{7,8}$ mass cluster ions but very few relate to 3-10 carbon atom systems. The present work addresses the fragmentation, in an inductively coupled rf plasma, of the tricyclic hydrocarbon adamantane and the significance of this with respect to film deposition.

The hydrocarbon, adamantane, and its triaza-analogue, hexamethylenetetramine as precursors are known ${ }^{2}$ to involve molecular fragmentations giving substantial abundances of relatively stable polyatomic clusters. As a result, faster deposition, different, or even better DAC-type films may result from such precursors under appropriately selected running conditions. In the course of the study of the mass-energy profile of the contents of an inductively coupled rf plasma, derived from the $\mathrm{Ar} / \mathrm{C}_{10} \mathrm{H}_{16}$ system, differently sized clusters of significant abundances were observed in the power range used (50-200 W at $13.56 \mathrm{MHz})$. These clusters, demonstrably, had reasonable stability/lifetimes, i.e., the abundances of various clusters observed change as the applied power, in the first instance, was switched manually, up or down in the indicated operating power range. For better control and convenience, a switchable power supply, offering a selected "ON/OFF" duty cycle at a given power level was designed and implemented. Using this, near selection of cluster abun- 


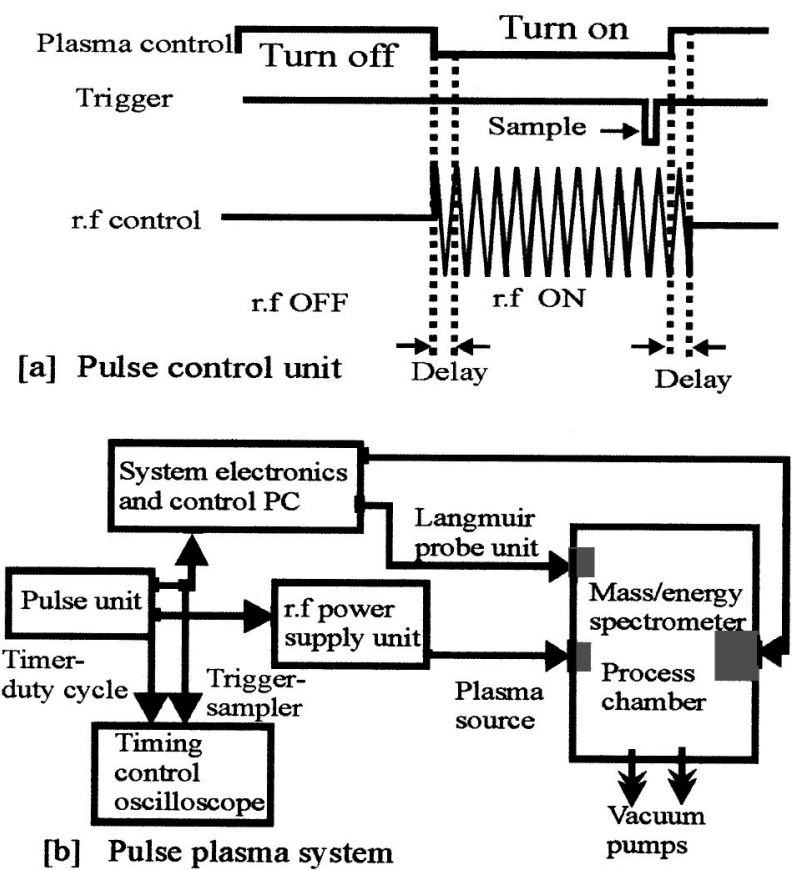

FIG. 1. Pulse plasma processing system. Simplified schematic of the pulse unit controlling the rf power supply which generates the rf plasma (top); overview of the plasma system during processing (bottom).

dance(s) for a chosen ion (or ions) is achievable. In consequence, films can be deposited, using a selected cluster or from a mixed- cluster regime where one or two clusters are dominant, without sophisticated mass-selected filtering. As described, successful selection of clusters has been achieved, using a pulsed rf inductively coupled plasma (ICP) under the deposition conditions outlined in Sec. II. The plasma contents were characterized by mass spectroscopy and Langmuir probe plasma diagnostics, while the deposited films were characterized by x-ray photoelectron spectroscopy, contact angle, and optical measurements.

\section{EXPERIMENT}

Adamantane, $\mathrm{C}_{10} \mathrm{H}_{16}$, as a precursor, fragments in the plasma to give clusters of significant size with reasonable stability/lifetimes in the user-defined running regime of the ICP source. The abundances of the various clusters generated are found $\mathrm{d}^{2}$ to change as the applied power is switched up or down in the 50-200 W rf power level range. Running the power supply in the switched mode, for a chosen power level, gas pressure, and flow, a power "on/off" ratio can be set so as to favor the presence of particular clusters in the plasmas. The pulse unit was designed and engineered inhouse, then integrated with the processing system. A simplified schematic explaining the operating mode of the pulse unit is shown in Fig. 1. The pulse unit was used to drive the plasma in a series of different pulsed on/off cycles by switching "on" and "off" the power delivered by the rf generator to the ICP source in user-defined, near square wave forms. The unit was also fitted with a programmable sample signal which can be switched by choice into the electronics of the diagnostic tools available (the mass-energy spectrometer or the Langmuir probe). This facility allows the triggering of
TABLE I. Summary of the XPS and contact angle $(\theta)$ data of the films deposited under $120 \mathrm{~W}, 6$ mTorr, using $\mathrm{C}_{10} \mathrm{H}_{16} / \mathrm{Ar}\left(\mathrm{D}_{01}-\mathrm{D}_{02}\right.$ and $\left.\mathrm{D}_{06}-\mathrm{D}_{07}\right)$ and $\mathrm{C}_{10} \mathrm{H}_{16} / \mathrm{N}_{2}\left(\mathrm{D}_{03}-\mathrm{D}_{05}\right.$ and $\left.\mathrm{D}_{08}-\mathrm{D}_{13}\right)$ gas mixtures and the following on/ off ratios: (a) $50 / 150 \mu \mathrm{s}$, (b) $150 / 50 \mu \mathrm{s}$, and (c) $180 / 20,20 / 180$ and $100 / 100$ $\mu$ s. Sample $\mathrm{D}_{00}$ is a blank sample showing 24.5 and 29.3 for $\mathrm{Si} 2 p$ and $\mathrm{Si} 2 s$, respectively.

\begin{tabular}{lcccccc}
\hline \hline Sample & $\begin{array}{c}\text { On/Off } \\
(\mu \mathrm{s})\end{array}$ & $\begin{array}{c}\mathrm{C} 1 s \\
(\text { at. \%) }\end{array}$ & $\begin{array}{c}\mathrm{N} 1 s \\
(\text { at. \%) }\end{array}$ & $\begin{array}{c}\text { O } 1 s \\
(\text { at. \%) }\end{array}$ & $\begin{array}{c}\text { Width } \\
(\mathrm{eV})\end{array}$ & $\begin{array}{c}\text { Contact angle } \\
\left({ }^{\circ}\right)\end{array}$ \\
\hline (a) & & & & & & \\
$\mathrm{D}_{01}$ & $50 / 150$ & 98.0 & - & 2.0 & 1.7 & 83 \\
$\mathrm{D}_{02}$ & $50 / 150$ & 98.0 & - & 2.0 & 1.7 & 84 \\
$\mathrm{D}_{03}$ & $50 / 150$ & 87.0 & 10.0 & 3.0 & 2.0 & 74 \\
$\mathrm{D}_{04}$ & $50 / 150$ & 86.0 & 8.0 & 6.0 & 2.0 & 72 \\
$\mathrm{D}_{05}$ & $50 / 150$ & 91.0 & 7.0 & 2.0 & 2.0 & 72 \\
$(\mathrm{~b})$ & & & & & & \\
$\mathrm{D}_{06}$ & $150 / 50$ & 86.0 & - & 14.0 & 1.8 & 68 \\
$\mathrm{D}_{07}$ & $150 / 50$ & 88.0 & - & 12.0 & 1.7 & 70 \\
$\mathrm{D}_{08}$ & $150 / 50$ & 70.0 & 17.0 & 13.0 & 2.5 & 67 \\
$\mathrm{D}_{09}$ & $150 / 50$ & 67.0 & 25.0 & 8.0 & 2.7 & 42 \\
$\mathrm{D}_{10}$ & $150 / 50$ & 67.0 & 30.0 & 3.0 & 2.8 & 41 \\
$(\mathrm{c})$ & & & & & & \\
$\mathrm{D}_{00}$ & - & 19.0 & - & 27.2 & - & 49 \\
$\mathrm{D}_{11}$ & $180 / 20$ & 58.0 & 38.5 & 3.5 & 3.3 & 34 \\
$\mathrm{D}_{12}$ & $20 / 180$ & 93.0 & 4.0 & 3.0 & 1.9 & 77 \\
$\mathrm{D}_{13}$ & $100 / 100$ & 72.0 & 25.0 & 3.0 & 2.7 & 64 \\
\hline \hline
\end{tabular}

the collection of diagnostic data at any user-defined point of the on/off duty cycle. ${ }^{9}$ A Hiden (Model: EPQ500) massenergy diagnostics system fitted to the vacuum vessel used in the deposition experiments was used to analyze the mass distributions of the cluster ions present in the pulsed plasma.

In the current work, we report on the plasma composition determined using the mass-energy diagnostic system and on thin-film specimens prepared using a $200 \mu$ s duty cycle, with the "on/off" periods $(\mu \mathrm{s})$ changed for the series $\mathrm{D}_{01}-$ $\mathrm{D}_{13}$ as follows: $\mathrm{D}_{01}-\mathrm{D}_{05}(50 / 150), \mathrm{D}_{06}-\mathrm{D}_{10}(150 / 50)$, $\mathrm{D}_{11}(180 / 20), \mathrm{D}_{12}(20 / 180), \mathrm{D}_{13}(100 / 100)$. Blank, unbiased surface-oxidized silicon coupons $\left(1-3 \mathrm{~cm}^{2}\right)$, typified by $\mathrm{D}_{00}$ $\left(\sim 2 \mathrm{~cm}^{2}\right)$, cleaned ultrasonically in redistilled iso-propanol just prior to deposition, were used as substrates. Note, the total duty cycle itself in all cases remained at $200 \mu \mathrm{s}$, and that " $x x / y y$ " means that the plasma is switched "on" for " $x x$ " $\mu$ s then "off" for " $y y$ " $\mu$ s throughout the 10 min deposition period used. Reliable control of the deposition regime requires a constant flow and a constant pressure of the precursors for running with the chosen fixed argon or fixed nitrogen flow, over an extended and controllable period of time. Therefore, to have better control of the precursor vapor, over realistic run times, the deposition system ${ }^{10,11}$ was fitted with a heated $\left(T \leqslant 110^{\circ} \mathrm{C}\right)$ vapor source (MKS 1150), gas mass-flow controllers (MKS 1179) and a main controller (MKS 247C). For the films discussed here, deposition was carried out at $120 \mathrm{~W}$ with a $6 \mathrm{mTorr}$ total gas/vapor mixture pressure at a $5 \mathrm{sccm}$ argon or nitrogen flow. The on/off duty cycle regimes switching the plasma on and off are summarized in Table I.

The Langmuir probe and the associated electronics used were also designed and developed ${ }^{12}$ in-house along with a suite of Windows-controlled acquisition and data reduction software. The pulse unit referred to above was fitted to the 
Langmuir probe in order to investigate the electrical characteristics of the plasma as a function of the various on/off regimes within the chosen duty cycle. The self-compensating Langmuir probe ${ }^{13}$ used consisted in this case of a thin tungsten wire appropriately positioned in the plasma. In practice, this probe, driven by a programmable dc voltage, is used to output the electrical characteristics of the plasma, i.e., bulk electron temperature $k T_{e}$, plasma potential $V_{p}$, floating voltage $V_{f}$, electron energy distribution (eed), and ion/ electron density $\left(N_{i}, N_{e}\right)$, etc. The current $I$ drawn by the probe is measured as a function of the driving voltage $V$ to extract an $I-V$ characteristic which is governed by the composition and the thermodynamic state of the plasma.

The $\mathrm{C}_{x} \mathrm{H}_{y}$ films $\left(\mathrm{D}_{01}-\mathrm{D}_{02}, \mathrm{D}_{06}-\mathrm{D}_{07}\right)$ and those containing nitrogen $\mathrm{C}_{x} \mathrm{~N}_{y} \mathrm{H}_{z}\left(\mathrm{D}_{03}-\mathrm{D}_{05}\right.$, and $\left.\mathrm{D}_{08}-\mathrm{D}_{13}\right)$, were prepared using the rf $(13.56 \mathrm{MHz})$ ICP system from the appropriate gas/vapor mixtures, $\mathrm{Ar} / \mathrm{C}_{10} \mathrm{H}_{16}$ or $\mathrm{N}_{2} / \mathrm{C}_{10} \mathrm{H}_{16}$, respectively. After deposition, $\mathrm{x}$-ray photoelectron spectroscopy (XPS) characterization was carried out ex situ in an XSAM800 (Kratos) system with a hemispherical energy analyzer $(\langle r\rangle=127 \mathrm{~mm}$, in the FAT mode with a pass energy of $38 \mathrm{eV}$ ), using nonmonochromatic $\operatorname{Mg} K_{\alpha}$ excitation (anode voltage $13 \mathrm{kV}$, at $18 \mathrm{~mA}, h \nu=1253.6 \mathrm{eV}$ ). The XPS system operated at $\sim 5 \times 10^{-10}$ Torr and was calibrated with $\mathrm{Ag} 3 d_{5 / 2}$, indicating a full width half maixmum of $1.3 \mathrm{eV}$. In order to facilitate the comparison of the specimens as a function of the deposition regime, namely the power applied, the on/off ratio, the composition (at. \%), width at half maximum of the $\mathrm{C} 1 s$ envelope, band shapes and chemical shifts are considered. Structural analysis of the XPS data involved curve fitting of $\mathrm{C} 1 s$ and $\mathrm{N} 1 s$ envelopes using a Gaussian line shape to give $\mathrm{CN}$ bonding patterns based on the models reported elsewhere. ${ }^{14}$

Following the XPS analysis, contact angle characterization was undertaken to further qualify the results obtained using an automated PC-controlled system (KSV Instruments Ltd.,CAM 200 model), operating a charge coupled device camera and frame store, through the comprehensive data acquisition and data reduction software provided. An accurate, volume-selectable, motorized microsyringe droplet dispenser, together with image capture (up to 25 frames/s) is included. Typically, with $5 \mu \mathrm{l}$ droplets, the reproducibility of the contact angle determined via the software is $\pm 0.5^{\circ}$. The contact angle ${ }^{15,16} \theta$ is defined as the interfacial tension between a solid, a liquid, and a vapor and is a measure of the surface energy of the solid surface underneath the microdrop. The form of the droplet and the resulting contact angle given by the tangent is illustrated in Fig. 2 as a function of the surface energy. Finally, the refractive index and the thickness of the deposited films were measured using an ellipsometer (Transtronics Ltd, Model MK. II), calibrated with a blank silicon sample and, equipped with a $6328 \AA$ wavelength laser (Novette, Model 150P-0) where the incident beam and the collection angles are at $70^{\circ}$, respectively.

\section{RESULTS AND DISCUSSION}

\section{A. Cluster selection}

Prior to the current work reporting on the cluster deposition of DAC and its nitrogen-containing analogues

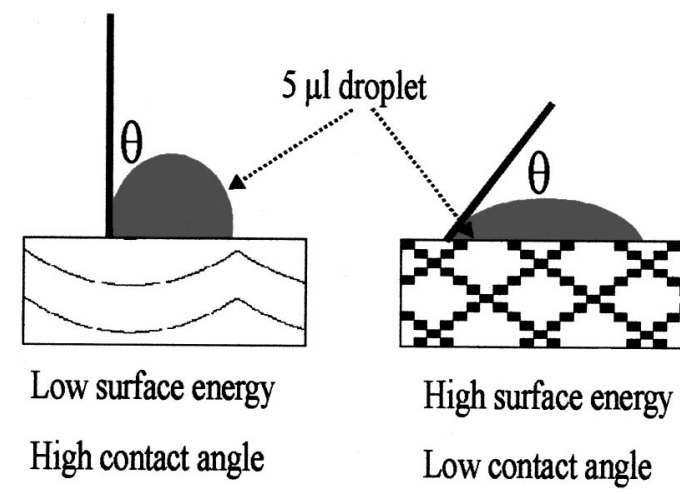

FIG. 2. Schematic showing the complementarity of surface energy and contact angle.

$\left(\mathrm{C}_{x} \mathrm{~N}_{y} \mathrm{H}_{z}\right)$, a systematic study was carried out to determine how the selected deposition regime affects the plasma. The power level of the rf plasma and the operating pressure play a dominant role in the fragmentation of adamantane in the inductively coupled source used, consequently on the plasma composition (and therefrom, the deposited films). The observed abundances of clusters of a particular size resulting from the fragmentation of adamantane molecule, is found to be a function of the power level and the processing gas makeup and pressure. For instance, at a low gas pressure $\sim 1$ mTorr, a higher power level will be favorable to the presence of low mass clusters (mass $\sim 26$ ) whereas, a lower power level will provide a larger cluster mass range, including clusters in significant numbers at high masses. Similarly, for a chosen power level, a low gas pressure will show low mass clusters in contrast to the behavior at higher pressures, giving cluster masses in the range of $12-136$. This is illustrated in Fig. 3(a) where the abundance and size of the clusters are related directly to the power level and gas pressure, e.g., at $150 \mathrm{~W}$ at $1 \mathrm{mTorr}$, the mass 26 cluster, $\mathrm{C}_{2} \mathrm{H}_{2}$, is dominant [Fig. 3(a1)] whereas additional species appear in a comparable proportion at $80 \mathrm{~W}$ and 1.3 mTorr [Fig. 3(a2)]. In turn, an increase of the pressure to 23 mTorr for $80 \mathrm{~W}$ [Fig. 3(a3)], induces a change in the cluster population, in the sense that a much higher abundance (intensity) appear for $\mathrm{C}_{7} \mathrm{H}_{9}^{+}$clusters (mass 93).

A significant operational factor, resulting from the observed stability of the cluster species in the plasma, is the switchable on/off ratio chosen to drive the plasma at a selected power level. For a chosen deposition regime, in terms of power level and operating pressure, flow etc., if the plasma is switched "on" and "off" to give a particular on/ off ratio, clusters of particular size appear preferentially under diagnostic mass analysis of the plasma contents. It should be noted that the plasma itself is not switched ON and OFF as the supply is driven, rather the cluster composition of the plasma changes because of the differing stabilities of the clusters present as the applied power is pulsed on and off. Figure 3(b) shows typical examples of how the on/off ratio influences the plasma contents, and Fig. 3(b3) gives the full mass spectrum of the adamantane molecule fragmented under $70 \mathrm{eV}$ electron energy impact, exhibiting clusters over the fragmentation range scanned. In this particular case, the 

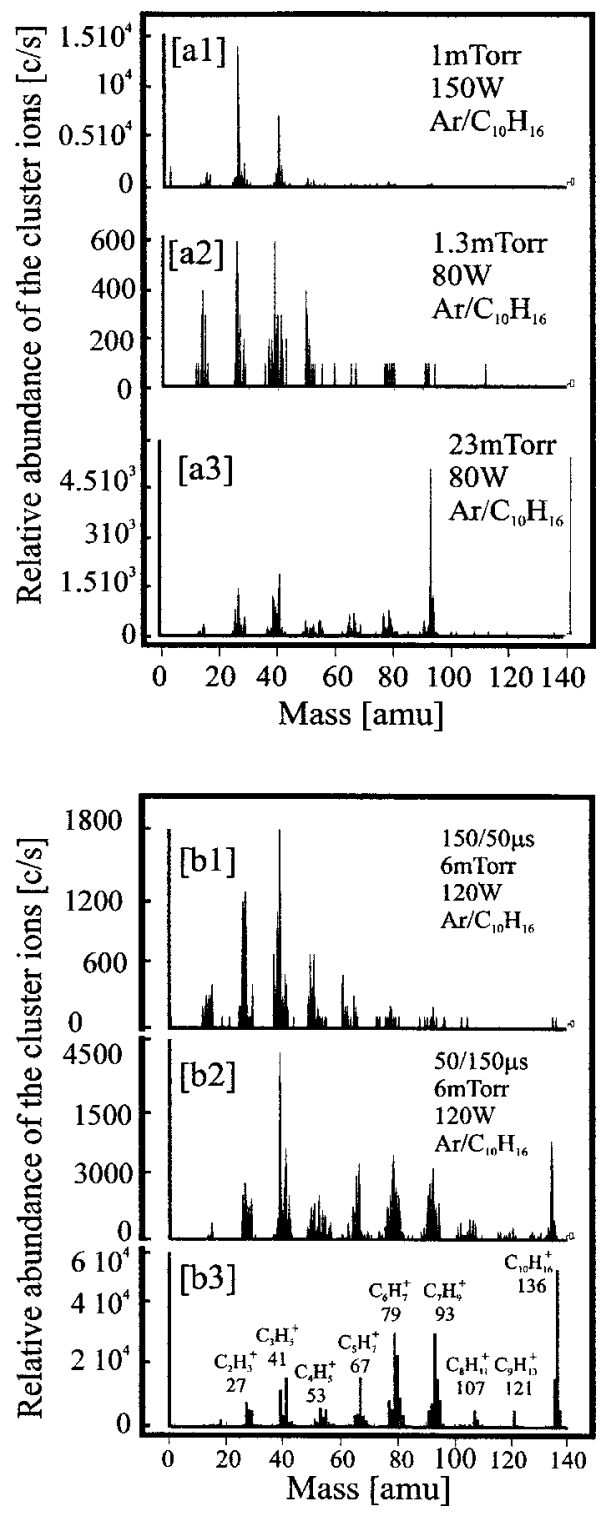

FIG. 3. Mass distribution of $\mathrm{C}_{10} \mathrm{H}_{16}$ fragmentation as a function of the experimental conditions. (a) Importance of the power level and gas pressure in the fragmentation of $\mathrm{C}_{10} \mathrm{H}_{16}$. (b) Importance of the on/off regimes (50/ 150 and $150 / 50 \mu \mathrm{s}$ ) in the fragmentation of $\mathrm{C}_{10} \mathrm{H}_{16}$. (b3) shows the fragmentation of $\mathrm{C}_{10} \mathrm{H}_{16}$ at $70 \mathrm{eV}$ electron impact, the reference spectrum.

plasma is "on" continuously. Figures 3(b1) and 3(b2), in turn, show the diagnostic mass spectra obtained running the plasma in the pulse mode, with 150/50 and 50/150 "on/off" duty cycles, respectively. It is clear that the $150 / 50$ ratio gives predominantly low mass clusters, notably 26 (and the argon ion at mass 40). The shorter the plasma "on" period, for example $50 \mu \mathrm{s}$ "on" and $150 \mu \mathrm{s}$ "off," the higher cluster masses appear. This is illustrated in Fig. 3(b2), with masses at 26, 41, 53, 67, 79, 93 and, now mostly, at 136. Higher masses are clearly more abundant in Fig. 3(b2), as opposed to their relatively low abundances in Fig. 3(b1).

In addition, Fig. 4 shows the fragmentation ratio on $\mathrm{N}_{2}^{+} / \mathrm{N}^{+}$as a function of the duty cycle where the "on" time varies from 20 to $200 \mu \mathrm{s}$. Note that above $40 \mu \mathrm{s}$ the $\mathrm{N}_{2}^{+} / \mathrm{N}^{+}$ ratio increases with the "on" time, whereas below $40 \mu$ s the relative intensity of the $\mathrm{N}^{+}$fragment is greater than that of

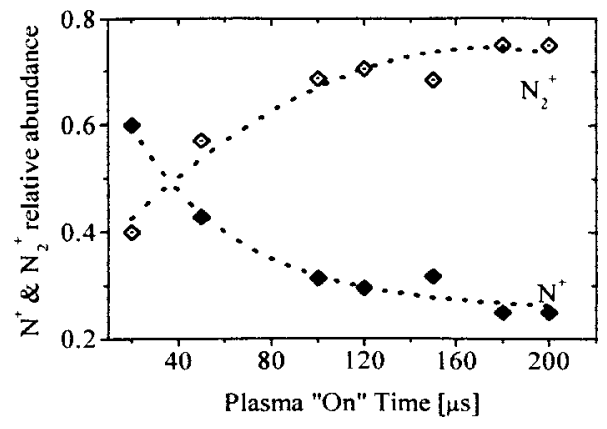

FIG. 4. Relative abundances of $\mathrm{N}_{2}^{+}$and $N^{+}$as a function of the on/off ratio.

$\mathrm{N}_{2}^{+}$. This inversion of $\mathrm{N}_{2}^{+}$to $\mathrm{N}^{+}$ratio from the low to higher "on" period may play a role in the deposition of nitrogenated DAC. By controlling the duty cycle of the plasma, we now have a means of fixing the $\mathrm{N}_{2}^{+} / \mathrm{N}^{+}$ratio during growth of DAC films and thereby allowing a better understanding of the growth mechanism.

\section{B. Plasma diagnostics}

All the electrical data extracted using the Langmuir probe were measured under $100 \mu$ s duty cycles (a) from a nitrogen plasma ( $30 \mathrm{mTorr}, 200 \mathrm{~W}$, and on/off $=50 / 50 \mu \mathrm{s})$ for the eed only and (b) from an argon plasma (50 mTorr, 50 W with the "on" time varying from 20 to $80 \mu \mathrm{s}$ ) for $k T_{e}$, $V_{p}, V_{f}, N_{e}$, and $N_{i}$. The latter parameters were measured at $20 \mu$ s into the rf "on" time because the rf power supply takes $\sim 10 \mu$ s to stabilize to the set. The eed functions were collected at $52 \mu$ s (just before the power switched off) and at $57 \mu \mathrm{s}$ (after the plasma has stabilized post rf switch off), in a 50/50 $\mu \mathrm{s}$ on/off sequence. The results of the plasma diagnostics are given in Fig. 5, showing that $k T_{e}, V_{p}$, and $V_{f}$ are functions of the on/off ratio. It also indicates that as the "on" increases, more energy is coupled into the plasma which transforms itself into plasma temperature [Fig. 5(a)]. Similar behavior is observed for $N_{e}$ and $N_{i}$ with $N_{i}>N_{e}$, [Fig. 5(b)] suggesting that the electrons are lost in the plasma faster than ions at the end of each "on" time. ${ }^{12}$ The eed measured at 52 and $57 \mu$ s trigger positions also exhibit clear differences, as expected [Fig. 5(c)]. Finally, the electrical characteristics of the plasma, under given conditions, such as power level, gas pressure, and makeup, can be monitored by pulsing the plasma at user-defined on/off regimes and data sampling positions. Controlling the cluster ion abundances and the plasma parameters could help in anticipating the properties of a surface treated under controlled conditions. The significance of the on/off ratio in regard to just what is then deposited follows.

\section{XPS analysis of cluster deposited films}

Different "on/off" regimes (Table I) have been chosen to generate the potentially different structural and chemical compositions of deposited films resulting from the varied abundances and cluster sizes present in the resultant plasmas. Two sets of specimens were considered, those films deposited using $\mathrm{Ar} / \mathrm{C}_{10} \mathrm{H}_{16}$ and those derived from $\mathrm{N}_{2} / \mathrm{C}_{10} \mathrm{H}_{16}$ mixtures, giving $\mathrm{C}_{x} \mathrm{H}_{y}$ (hydrogenated DAC), and the analogous 

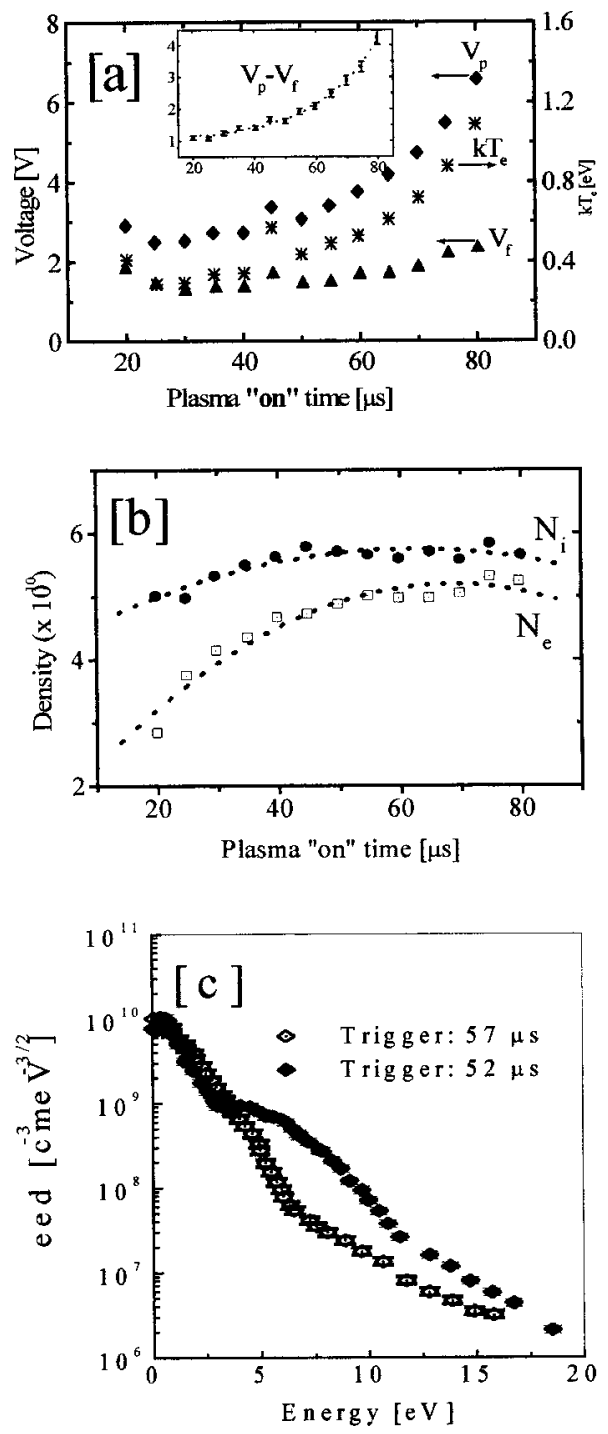

FIG. 5. Electrical characteristics of the plasma recorded using Langmuir probe $V_{p}, V_{f}, k T_{e}$ (a) were collected in an argon plasma with $50 \mathrm{mTorr}, 50$ $\mathrm{W}$ as a function of the "on" time, whereas electron and ion density (b) were collected under the same conditions but with $20 \mu$ s trigger offset. The eed (c) was measured in a nitrogen plasma (200 mTorr) at the trigger positions 52 and $57 \mu \mathrm{s}$.

$\mathrm{C}_{x} \mathrm{~N}_{y} \mathrm{H}_{z}$ (carbon nitride films), respectively. The DAC-type specimens $\left(D_{01}-D_{02}\right.$ and $\left.D_{06}-D_{07}\right)$ exhibit almost identical XPS spectra with repeated features in the $\mathrm{C} 1 \mathrm{~s}$ band envelopes regardless of the on/off regime, with the other deposition parameters kept constant. However, small differences are present. For instance, 150/50 ratio films show a slightly broader band with a nominal width at half maximum of 1.8 $\mathrm{eV}$ against $1.7 \mathrm{eV}$ for that from the 50/150 regime. Additionally, the amount of oxygen present (via XPS survey scans) at the surface of these films is relatively higher in the 150/50 regime at $\sim 12$ at. $\%$ against $\sim 2$ at. $\%$ for the $50 / 150$ case. This is probably related to the difference in the energy generated by the two regimes, whereby higher energy in the plasma generates relatively porous structures. Note, however, the oxygen presence here remains at the superficial contamination level. ${ }^{17}$
The second set $\left(D_{03}-D_{05}\right.$ and $\left.D_{08}-D_{13}\right)$ of specimens refers to the samples containing nitrogen deposited under the varied duty cycles above in Table I. Initially, samples deposited under switched on/off regimes of 50/150 and 150/50 $\mu$ s are considered. XPS analysis of the specimens deposited under these regimes now show significant differences, as listed in Table I. Generally, these are identified in terms of atomic concentration, envelope shapes, and chemical shifts resulting solely from the change of the on/off ratio. Specimens deposited under a 50/150 on/off ratio show very little oxygen at the surface (2-6 at. \%), whereas those deposited at $150 / 50$ contain more oxygen (3-14 at. \%) as in the previous nonnitrogenated samples. Assuming the oxygen level is due to superficial contamination and since the experiments were carried out under similar quality vacuum conditions (chamber base pressure $10^{-9}$ Torr), these oxygen levels in the films suggest that samples deposited at 150/50 are apparently more likely to absorb or accept oxygen at the outmost layers than are the 50/150 films. Comparison of Table I(a) and (b) also shows differences in terms of the carbon and nitrogen present in these films. Very little nitrogen is found in the samples deposited at a 50/150 ratio [7-10 at. \%, Table I(a)], whereas, in those grown under 150/50 significantly more nitrogen is found [17-30 at. \%, Table I(b)]. These differences in both $\mathrm{N}$ and $\mathrm{O}$ may probably be due to a similar reason and related to the uptake of "impurities" by DAC films by virtue of its porous microstructure controlled by the energy of the depositing species.

Table I(a) and (b) also summarizes the nominal width at half maximum of the XPS C $1 s$ envelopes as a function of the atomic concentration. The specimens grown at a 50/150 on/off ratio present narrower, more symmetrical, regular band profiles, with the peak positions remaining identical [Fig. 6(a)]. The width of $\mathrm{C} 1 s$ envelope varies from $1.7 \mathrm{eV}$ for the DAC-type samples to $2.0 \mathrm{eV}$ for the nitrogen containing specimens [Table I(b)]. In turn, samples deposited under a 150/50 regime give widths of $1.7-1.8 \mathrm{eV}$ for the DAC-type films (similar to the previous set) and of $2.5-3.0 \mathrm{eV}$, as function of nitrogen level for the nitrogen containing samples. As expected, the latter set of samples show broader and nonsymmetrical band shapes as the nitrogen level increases in the films [Fig. 6(a), Table I(b)]. The superposition of Figs. $6(\mathrm{a})$ and $6(\mathrm{~b})$ indicate identical band positions for the DACtype sample regardless of the on/off ratio used. In turn, a slight chemical shift $(\sim 0-1 \mathrm{eV})$ appears in the nitrogencontaining films when the on/off ratio is varied from 50/150 to $150 / 50 \mu \mathrm{s}$. This shift increases with an increasing nitrogen level in the films, likewise associated with the increasing band asymmetry. ${ }^{18}$ This suggests the presence of $\mathrm{C}-\mathrm{N}$, $\mathrm{C}=\mathrm{N}$, and $\mathrm{C} \equiv \mathrm{N}$ components but also a possible formation of polymeric structures. ${ }^{19}$

Given the role of the on/off ratio in determining the plasma contents, Fig. 6, as well as the deposition process, another set of experiments was carried out to confirm and qualify the earlier results presented above, i.e., that the atomic abundance is a function of the on/off ratio. Three series of samples were deposited under 180/20, 20/180, and $100 / 100 \mu \mathrm{s}$ on/off ratios. Representative results are summarized in Table I(c) and Fig. 6(c). The same trends in atomic 

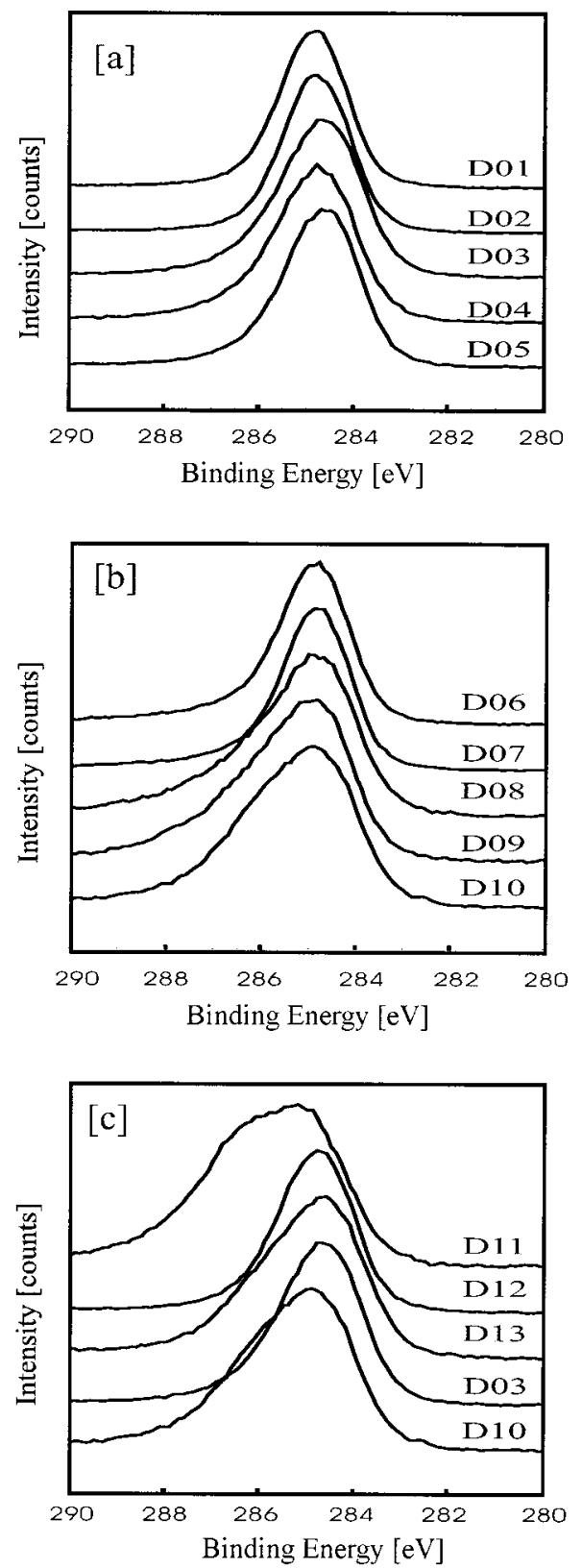

FIG. 6. Comparison of the XPS C $1 s$ envelopes of the specimens grown under the deposition regimes summarized in Table I: 50/150 $\mu \mathrm{s}$ (a), 150/50 $\mu$ s (b), 180/20, 20/180 and 100/100 $\mu$ s (c).

concentration, width at half maximum, band shape, and chemical shift are confirmed. The oxygen contents remain at a contamination level $\sim 3$ at. $\%$ in this set but the nitrogen concentration rises to $\sim 38$ at. \% for the highest "on" period $(180 / 20 \mu \mathrm{s})$ and is the lowest at $\sim 4$ at. \% for the reverse regime $(20 / 180 \mu \mathrm{s})$, with the $\mathrm{C} 1 s$ envelope widths at half maximum 3.3 and $1.9 \mathrm{eV}$, respectively. The 100/100 regime data further confirm expectation, with the nitrogen present in the resulting films at $\sim 25$ at. \%, with $2.7 \mathrm{eV}$ for the $\mathrm{C} 1 \mathrm{~s}$ bandwidth at half maximum showing a regular and symmetrical shape. It can be concluded, therefore, that the longer the power pulse stays on the more nitrogen is present in the films derived from a $\mathrm{N}_{2} / \mathrm{C}_{10} \mathrm{H}_{16}$ mixture. The opposite is also true in the sense that the nitrogen level in the films is lower if the power is switched on for a significantly shorter period
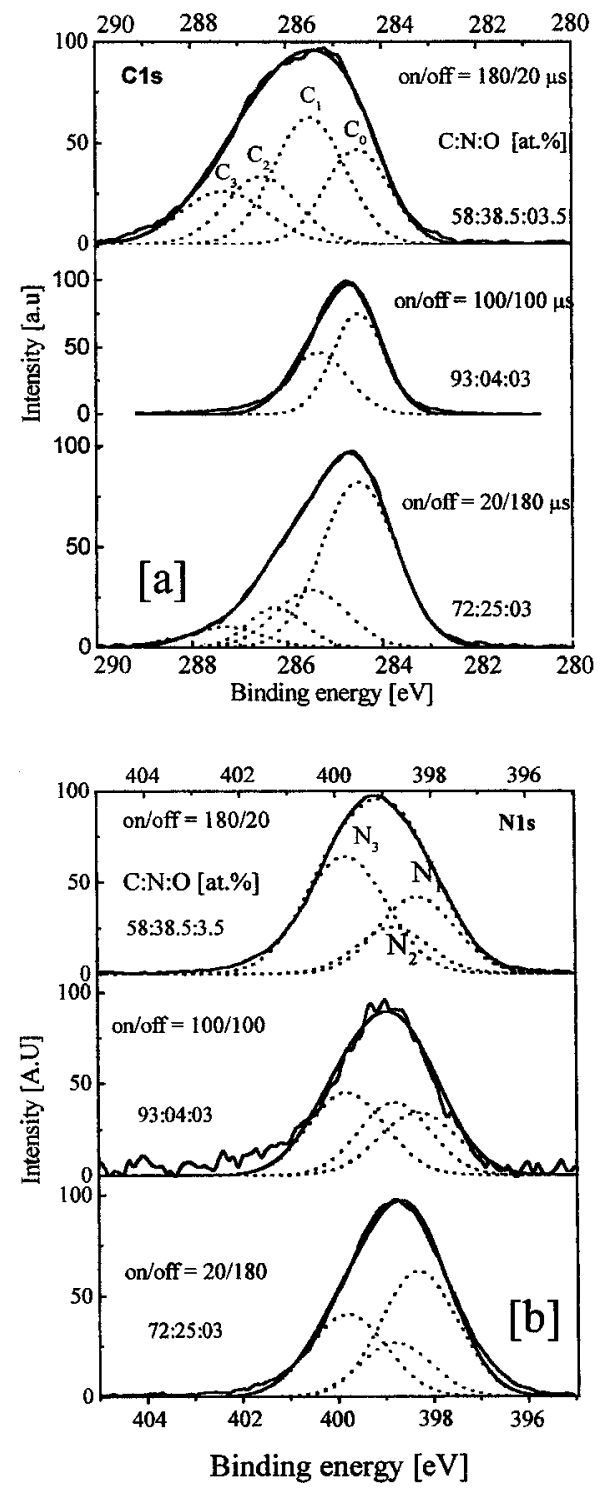

FIG. 7. Curve fitting of the $\mathrm{C} 1 s$ (a) and $\mathrm{N} 1 s$ (b) envelopes for samples deposited under 20/180, 100/100, and 180/20 $\mu$ s on/off regimes, indicating the variation in the microstructures $\left(s p / s p^{2} / s p^{3}\right)$ as a function of the deposition regime.

in the duty cycle. As a result, a 1:1 on/off ratio produces nitrogen-containing films with a near median proportion of nitrogen in the films. Referring to the data presented in Fig. 4 , showing a high abundance of $\mathrm{N}_{2}^{+}$at the longer "on" periods, one can assume that $\mathrm{N}_{2}^{+}$plays a significant role in the formation of $\mathrm{C}_{x} \mathrm{~N}_{y} \mathrm{H}_{z}$ compounds, although additional data may be required before a final conclusion is drawn on the incorporation of nitrogen in hydrogenated amorphous carbon matrix. The energetic $\mathrm{N}_{2}^{+}$species may also play a role as an energetic etchant of weaker $s p^{2}$ bonds, which then allows high absorption within the film microstructure.

Curve fitting of $\mathrm{C} 1 s$ and $\mathrm{N} 1 s$ envelopes of specimens $D_{11}, D_{12}$, and $D_{13}$ is given in Fig. 7, indicating the change in the microstructure as a result of the deposition regime. By analogy to fitting data published, ${ }^{14} \mathrm{C}_{1}-\mathrm{C}_{4}$ and $\mathrm{N}_{1}-\mathrm{N}_{3}$ are attributed to $\mathrm{C}-\mathrm{C} / \mathrm{C}=\mathrm{C}(284.5 \mathrm{eV}), \quad \mathrm{C}=\mathrm{N}(285.5 \mathrm{eV})$, $\mathrm{C}-\mathrm{N}(287.7 \mathrm{eV}), \mathrm{C} \equiv \mathrm{N}(286.8 \mathrm{eV})$, and $\mathrm{N}-\mathrm{C}(398.3 \mathrm{eV})$, $\mathrm{N}=\mathrm{C}(399.9 \mathrm{eV}), \mathrm{N} \equiv \mathrm{C}(398.8 \mathrm{eV})$, respectively. Significant 
oxygen contribution to the $\mathrm{C} 1 s$ and $\mathrm{N} 1 s$ envelopes was discounted because very little oxygen $(\sim 3$ at. \%) was found in the specimens considered. Clearly, the operating duty cycle and the on/off ratio are significant factors unequivocally influencing the contribution of $s p / s p^{2} / s p^{3}$ fractions to the $\mathrm{C} 1 s$ and $\mathrm{N} 1 s$ envelope. Figures 7(a) and 7(b) show that the intensity of the band at $284.5 \mathrm{eV}$ increases as the "on" period decreases, giving $\mathrm{C} 1 s$ centered at $285 \mathrm{eV}$ and $\mathrm{N} 1 s$ centered at $398 \mathrm{eV}$, indicative of amorphouslike-films. It is therefore reasonable to argue in agreement with those reports ${ }^{20,21}$ that establish a direct link between carbon clusters and the properties of the corresponding deposited films. In order to obtain additional structural information, contact angle measurements again were carried out.

\section{Contact angle measurements}

Contact angle $(\theta)$ measurements were performed on the same specimens in order to investigate possible structural or compositional differences on the film surfaces, reflected in changes in their surface energies as a result of the changes in the on/off ratio. A blank silicon wafer giving $\theta=49^{\circ}$ (distilled water, $18{ }^{\circ} \mathrm{C}$ ) is taken as a reference. The results are listed in Table I. Again DAC-type and nitrogen-containing samples are considered. The DAC-type specimens give a very high contact angle $\left(\theta>70^{\circ}\right)$ regardless of the on/off ratio used for deposition. In turn, nitrogen-containing samples show varying contact angle data depending on the duty cycle. Although DAC-type specimens all show high measured contact angles, some differences do occur. Indeed, comparison of the contact angles associated with the on/off ratios used indicates that clear differences exist. For instance, DAC-type films grown under a $150 / 50$ ratio give $\theta=70^{\circ}$, whereas those grown under a 50/150 on/off regime give $\theta=80^{\circ}$, suggesting enhanced tribological properties ${ }^{22}$ may be present in these cases, i.e., a low surface energy improves lubrication of an overcoat layer and reduces friction when used as a hard disk coating. Indeed, surface energy, contact angle, and lubrication are linked together ${ }^{23,24}$ and are of importance in magnetic storage technology. One aspect of pulsing the plasma in the context of film growth which is of interest is to control the resultant contact angle of the surfaces produced, leading for example to the use of much thinner lubricant layers when the film surface energy is very low. However, it should be noted that such high values in the contact angle may be indicative of smoother, flatter, and pin hole free films but could also suggest the presence of either a more amorphous or polymer-like with low hardness.

The XPS data shows that all samples deposited under a $50 / 150 \mu \mathrm{s}$ on/off ratio contain relatively little nitrogen. The corresponding contact angles are relatively high $\left(\theta>70^{\circ}\right)$, similar to those shown by the hydrogenated DAC-type specimens. The contact angles resulting from samples grown under a 150/50 ratio appear to depend on the nitrogen content, giving the highest value at $67^{\circ}$, with $\sim 17$ at. $\%$ nitrogen and $\sim 13$ at. \% oxygen present in the film. As the nitrogen content increases a significant decrease in the contact angle takes place. For instance, the contact angle decreases to $41^{\circ}$ when the nitrogen content increases to $\sim 30$ at. $\%$, with $\sim 3$ at. $\%$

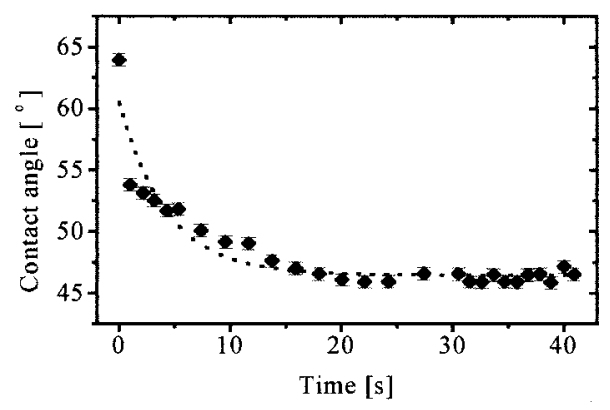

FIG. 8. Typical contact angle measurement of the specimens grown with $150 / 50 \mu$ s pulsed plasma in a $\mathrm{C}_{10} \mathrm{H}_{16} / \mathrm{N}_{2}$ gas mixture, as function of time.

oxygen. Note that the contact angles for samples $\mathrm{D}_{09-10}$ stabilize at a relatively low value although the initial value is high. Figure 8 illustrates a typical example with the water droplet spreading across the surface. 40 different frames were recorded at $1 \mathrm{~s}$ intervals. The contact angle is observed to decrease rapidly in the first few seconds then more slowly until a stable value is reached. The droplet $(5 \mu \mathrm{l})$ was found to stabilize after $\sim 15 \mathrm{~s}$ to give $\theta$ in the $65^{\circ}-40^{\circ}$ range for nitrogenated specimens grown under a $150 / 50$ on/off regime. In contrast, those grown under a 50/150 ratio conditions gave a near stable droplet behavior almost immediately, enabling repeated and near identical contact angle measurements to be made over extended periods of time (evaporation effects are discounted). These differences may be indicative of the surface stability or short-term instability, surface polarity of the films concerned but at some extent strongly suggest the presence of porous structures in the films.

On the basis of the XPS results obtained and the contact angle measurements, further measurements were performed on samples $\mathrm{D}_{11-12}$ grown under the conditions described earlier. As the nitrogen level increases to $\sim 38.5$ at. $\%\left(D_{11}\right.$, $180 / 20$ on/off ratio), the contact angle decreases significantly to $34^{\circ}$, following the trend already illustrated in Fig. 8. As expected, specimen $\mathrm{D}_{12}$ gave straightforward and stable contact angle data at $\sim 77^{\circ}$, consistent with the results presented in Table $\mathrm{I}(\mathrm{a})$. Specimen $\mathrm{D}_{13}$, grown under a $100 / 100 \mu$ s duty cycle, gives a stable contact angle $\left(64^{\circ}\right)$ which was measured over an extended period. Additionally, this sample contains $\sim 25$ at. $\%$ nitrogen and $\sim 3$ at. $\%$ oxygen, suggesting that a stable surface sample, offering good tribological properties, can be prepared by controlling the growth parameters carefully. The decrease in the contact angle cannot be attributed only to the presence of nitrogen since a high contact angle surface can be produced, providing an appropriate on/off regime is used for film deposition, i.e., structural, as well as compositional effects need to be considered.

\section{E. Optical data}

Optical refractive index measurements performed on the samples are shown in Table II. Specimens in Table II were selected to be a consistent representation of the various on/ off regimes used. The film thickness values obtained after 10 min deposition are consistently comparable with the growth rate of $0.6 \mu \mathrm{m} / \mathrm{h}$ reported previously ${ }^{11}$ for films prepared under similar, but nonpulsed, deposition conditions. The film 
TABLE II. Film thickness and refractive index as a function of the on/off regimes used.

\begin{tabular}{lccccc}
\hline \hline Specimens & $\mathrm{D}_{03}$ & $\mathrm{D}_{10}$ & $\mathrm{D}_{11}$ & $\mathrm{D}_{12}$ & $\mathrm{D}_{13}$ \\
\hline On/off regime $(\mu \mathrm{s})$ & $50 / 150$ & $150 / 50$ & $180 / 20$ & $20 / 180$ & $100 / 100$ \\
Refractive index & $2.08 \pm 0.04$ & $2.55 \pm 0.10$ & $2.56 \pm 0.09$ & $1.91 \pm 0.09$ & $2.37 \pm 0.08$ \\
Film thickness $(\AA)$ & $1271 \pm 36$ & $874 \pm 50$ & $870 \pm 42$ & $1457 \pm 100$ & $1017 \pm 60$ \\
\hline \hline
\end{tabular}

thickness and refractive indices listed in Table II indicate clearly the differences occurring as a consequence of the changing deposition regimes used. For instance, lower "on" regimes give a relative low refractive index and thicker films $\left(\mathrm{D}_{03}, \mathrm{D}_{12}\right.$, with $2.1,1270 \AA$ and $1.9,1460 \AA$, respectively). In turn, $150 / 50$ and $180 / 20 \mu$ s regimes exhibit near identical refractive index $(\sim 2.6)$ and film thickness $(870 \AA)$. It is anticipated that these results suggest a faster growth rate $(5: 3)$ for the lower "on" period (20/180 $\mu$ s) as opposed to longer "on" regime $(180 / 20 \mu \mathrm{s})$. Generally, the thickness should not affect the refractive index unless ultrathin films are considered. However, films with low refractive index exhibit a wider band gap while a narrow band gap is associated with a higher refractive index..$^{25}$ The films are transparent and therefore we expect them to exhibit a relatively high refractive index (1.9-2.6) as shown in Table II. It is believed that nitrogen incorporation in amorphous carbon films helps to promote the formation of $s p^{2}$ bonds followed by an increase in the refractive index and a decrease of the optical band gap as the film becomes more graphiticlike. The results presented here agree with those reported in the literature, ${ }^{26}$ which show a clear decrease of the optical band gap as the nitrogen concentration increases, suggesting an increase of refractive index as seen in Table II. These results once again indicate that optical properties of the amorphous carbon films can be controlled using appropriate pulse regimes, consistent with our earlier results regarding the surface chemistry, the structural, electrical, and tribological properties.

\section{F. General discussion}

Badyal et al. ${ }^{27}$ recently underlined the interest in the "smart surfaces" 28 by control of the deposition plasma. Pulsing a plasma has proved to be an efficient technique in surface polymerization ${ }^{29}$ processes and for enhancing adhesive treatment, appearing to allow better control of the desired surface properties. In a number of ways, the results presented in this article are in agreement with the work of Badyal, ${ }^{27}$ Hutton et al. ${ }^{28}$ and Ryan et al. ${ }^{29}$ while opening new application potential in thin film deposition for control of surface structure, morphology, and properties via a pulsed plasma. By adjusting the $s p^{2} / s p^{3}$ ratio, optical, electronic, ${ }^{30}$ and tribological properties ${ }^{31}$ of DAC-type carbons can be modified at will. For example, the band gap varies with the $s p^{2}$ clusters size and the conductivity with the $s p^{2}$ states. ${ }^{32}$ As such, tuning the mass distribution by pulse plasma control during film deposition therefore offers a powerful way of controlling film properties.

\section{CONCLUSION}

It has been demonstrated that hydrocarbon clusters, derived from the hydrocarbon $\mathrm{C}_{10} \mathrm{H}_{16}$, can be produced in an inductively coupled plasma and their size and relative abundances controlled, by choosing carefully the power on/off ratios for a fixed duty cycle generated by an adjustable switching unit in the driving rf supply. Mass-energy diagnostics show that smaller-sized clusters are produced when the plasma is switched on longer for the chosen duty cycle (200 $\mu \mathrm{s}$ in the current study), whereas the opposite on/off regime will produce larger-sized clusters preferentially. Langmuir probe diagnostic data from the plasmas indicate that the electrical characteristics observed are affected strongly by the experimental conditions, notably by the on/off switching regime and by the position of the trigger signal initiating data collection.

Postdeposition XPS data of the specimens grown under the duty cycles $(50 / 150,150 / 50,20 / 180,180 / 20$, and 100/ $100 \mu \mathrm{s})$ clearly indicate that the samples grown at shorter on periods contain very little nitrogen (up to 10 at. \%) and the oxygen remains at a superficial contamination level (2-6 at. \%). The $\mathrm{C} 1 s$ envelopes of these specimens show a regular shape and narrow envelope widths at half maximum (1.7$2.0 \mathrm{eV}$ ). In turn, specimens grown under longer "on" periods give broader envelopes (widths at half maximum $=1.8-3.3$ $\mathrm{eV}$, depending on the gas mixtures used for deposition) with asymmetrical band shapes. The latter regimes are favorable to a higher incorporation of nitrogen in the films $(\sim 38.5$ at. $\%$ for samples deposited under a 180/20 $\mu$ s on/off ratio) while the oxygen content is slightly higher $(\sim 3-12$ at. \%), although still at the contamination level. Specimens grown under 50/150, 20/180 $\mu$ s regimes gave high contact angles $\left(\theta=70^{\circ}-84^{\circ}\right)$, suggesting low surface energy with possibly better lubricant properties and low friction, as a result. In contrast, specimens deposited under the reverse ratios $(150 / 50$ and $180 / 20 \mu \mathrm{s})$ showed low contact angles, suggesting poorer tribological properties for such films. The significant decrease of the contact angle may be affected by any surface texture present. In these circumstances the possible porosity of the surface should be also considered. Finally, the combination of data derived from both XPS and contact angle measurements are consistent and suggest clear differences in the morphology (now under investigation), the stability, porosity, and polarity of such surfaces. Deposition dependent differences in the surface features of the processed films were further confirmed by the optical data showing lower refractive index and faster growth rate in the conditions described. The methodology used is now being refined and extended to other molecular precursors. 


\section{ACKNOWLEDGMENTS}

This work was carried out in the Surface Science Laboratory at the University of Ulster, Northern Ireland. The authors thank Seagate Technology Ireland, the Northern Ireland Research and Technology Unit (IRTU) and the University of Ulster for financial support. The authors also thank colleagues from the University of Surrey, Guildford, England for helpful discussions.

${ }^{1}$ A. Y. Liu and M. L. Cohen, Phys. Rev. B 41, 10727 (1990).

${ }^{2}$ R. Crawford, D. Philosophy thesis, University of Ulster, 1998.

${ }^{3}$ P. Melinon et al., Carbon 32, 1011 (1994).

${ }^{4}$ F. J. Roux, B. Cabaud, G. Fuchs, D. Guillot, A. Hoareau, and P. Melinon, Appl. Phys. Lett. 64, 1212 (1994).

${ }^{5}$ N. L. Clipston, T. Brown, Y. Y. Vasil'ev, M. P. Barrow, R. Herschuh, U. Reuter, A. Hirch, and T. Drewello J. Phys. Chem. 104, 9171 (2000).

${ }^{6}$ V. Paillard, P. Melinon, J.-P. Perez, V. Dupuis, A. Perez, J.-L. Loubet, H. Pascal, A. Tonck, and M. Fallavier, Nanostruct. Mater. 4, 759 (1994).

${ }^{7}$ D. Tanaka, Y. Ohkawara, N. Itoh, S. Ohshio, H. Ito, and H. Saitoh, Jpn. J. Appl. Phys., Part 1 39, 4148 (2000).

${ }^{8}$ M. S. Deleuze, M. G. J-P. Francois, and L. S. Cederum, J. Chem. Phys. 112, 325 (2000).

${ }^{9}$ D. A. Zeze, N. M. D. Brown, A. M. Joyce, and C. A. Anderson, Appl. Phys. Lett. 80, 22 (2002).

${ }^{10}$ D. A. Zeze, D. R. North, N. M. D. Brown, and C. A. Anderson, Surf. Interface Anal. 29, 201 (2000).

${ }^{11}$ D. A. Zeze, E. P. O'Toole, R. I. Crawford, N. Cui, C. A. Anderson, and N. M. D. Brown, Surf. Interface Anal. 26, 896 (1998).

${ }^{12}$ A. M. Joyce, PhD thesis, University of Ulster, 2000.
${ }^{13}$ J. V. Scanlon and M. B. Hopkins, J. Vac. Sci. Technol. A 10, 1207 (1992).

${ }^{14}$ S. Bahattacharyya, J. Hong, and G. Turban, J. Appl. Phys. 83, 3917 (1998); S. Bahattacharyya, C. Vallee, C. Cardinaud, and G. Turban, Diamond Relat. Mater. 8, 586 (1999).

${ }^{15}$ M. J. Jaycock and G. D. Parfitt, Chemistry of Interfaces (Ellis Horwood, 1981).

${ }^{16}$ H. Sakai and T. Fujii, J. Colloid Interface Sci. 210, 152 (1999).

${ }^{17}$ S. Lopez, H. M. Dunlop, M. Benmalek, G. Tourillon, M.-S. Wong, and W. D. Sproul, Surf. Interface Anal. 25, 827 (1997).

${ }^{18}$ A. Mansour and D. Ugolini, Phys. Rev. B 47, 10201 (1993).

${ }^{19}$ T. W. Scharf, R. D. Ott, D. Yang, and J. A. Barnard, J. Appl. Phys. 85, 3142 (1999).

${ }^{20}$ G. Pascoli and A. Polleux, Astron. Astrophys. 359, 799 (2000).

${ }^{21}$ E. Riedo, E. Magnano, S. Rubini, M. Sancrotti, E. Barborini, P. Piseri, and P. Milani, Solid State Commun. 116, 287 (2000).

${ }^{22}$ Li Yufeng and V. Sharma, J. Tribol. 122, 293 (2000).

${ }^{23} \mathrm{Ji}$-Go Zhang, Proceeding of IEEE Holm Conference on Electrical Contacts, New York, 1944, p. 145.

${ }^{24}$ J. Wu and C. M. Mate, Langmuir 14, 4929 (1998).

${ }^{25}$ R. U. A. Khan, A. P. Burden, S. R. P. Silva, J. M. Shannon, and B. J. Sealy, Carbon 37, 777 (1999).

${ }^{26}$ Y. H. Yu, Z. Y. Chen, E. Z. Luo, W. Y. Cheung, J. P. Zhao, X. Wang, J. B. Xu, S. P. Wong, and I. H. Wilson, J. Appl. Phys. 87, 2874 (2000).

${ }^{27}$ J. P. S. Badyal, Chem. Br. 2001, 45.

${ }^{28}$ S. J. Hutton, J. M. Crowther, and J. P. S. Badyal, Chem. Mater. 12, 2282 (2000).

${ }^{29}$ E. R. Ryan, A. M. Hynes, and J. P. S. Badyal, Chem. Mater. 8, 37 (1996).

${ }^{30}$ R. U. A. Khan and S. R. P. Silva, Diamond Relat. Mater. 10, 224 (2000).

${ }^{31}$ Y. Lifshitz, S. R. Kasi, J. W. Rabalais, and W. Eckstein, Phys. Rev. B 41, 10468 (1990).

${ }^{32}$ J. Robertson and E. P. O’Reilly, Phys. Rev. B 35, 2946 (2987). 